registrars to allow the non-medical professionals to gain necessary experience and competence.

Measurement of improvement The non-medical professionals needs training to be able to manage the required tasks autonomously, before the mapping process can be repeated.

Effects of changes We anticipate that training of the non-medical workforce to perform more advanced clinical tasks will result in more efficient patient care, with better provision of training and education for those existing trainees.

Lessons learnt A post-task debrief identified a degree of anxiety that the process was being used as a performance assessment tool. This suggests discordance between participants' performance and their declarative knowledge of the task. Another challenge was "mapping fatigue" that became particularly evident as the project progressed. Social acceptability determinants of self reporting affects the validity of data and to ensure the integrity of the data, data collection by independent observers may be a more effective method.

Message for others Diversifying the workforce is inevitable for paediatric departments in the future. The multi professional task analysis we undertook is a pathfinder for other organisations who will need to undergo similar change. While multi professional workforce use is well developed in other areas, there has to be recognition of the time required for non-medical professionals to develop the breadth of knowledge and skills required to function autonomously at middle grade level in general paediatrics. Targeted training would speed up the development process.

\section{G566(P) CHECKLISTS FOR TIME-CRITICAL EQUIPMENT FAILURE DURING PATIENT TRANSPORT}

${ }^{1} \mathrm{RJ}$ Kelly, ${ }^{1} \mathrm{VJ}$ Monnelly, ${ }^{2} \mathrm{~B}$ Stenson. ${ }^{1}$ Neonatal Transport, ScotSTAR - South East Scotland Division, Edinburgh, UK; ${ }^{2}$ Neonatology, NHS Lothian, Edinburgh, UK

10.1136/archdischild-2015-308599.515

Context Time critical faults with equipment can and have occurred during patient transport.

Problem Equipment failure can be a major problem during transport as spare equipment is not available in a timely fashion. It is essential to rapidly identify and resolve the problem to avoid patient harm.

Assessment of problem and analysis of its causes Previously our transport team relied on the clinical experience of staff to diagnose the fault, as well as "Quick Guides" for equipment. These "Quick Guides" are often up to 10 pages long and can be difficult to utilise effectively in a stressful situation.

Intervention We drew on the experience of other professionals who also encounter time critical faults. Pilots utilise emergency procedure checklists provided by the Federal Aviation Administration, which supply an immediate action sequence to be followed during emergency situations.

The systematic approach used by pilots (one pilot concentrates on maintaining flight through their Aviate / Navigate / Communicate approach, whilst another runs the checklist in an attempt to diagnose the fault) could be transferable to a medical setting (where one team member concentrates on maintaining patient stability through the Airway / Breathing / Circulation approach, whilst another runs the checklist in an attempt to diagnose the fault).

We produced single page checklists for each of the three different types of ventilator used by our service (see example below). After positive feedback from staff, we are now producing checklists for therapeutic hypothermia failure and nitric delivery system failure.

Study design Checklists are produced by staff concentrating on their area of expertise, and are checked by senior staff members and with equipment manufacturers where appropriate. Satisfaction with the use of checklists was assessed by survey and their ongoing use will be reviewed during simulator sessions.

Strategy for change Checklists have been used during staff training days, to provide an opportunity for staff to familiarise themselves with them, and to receive feedback about their use. Relevant checklists have now been attached to each transport trolley, for reference in emergencies. Staff from other transport teams have now asked for copies to develop their use within their service.

Measurement of improvement Satisfaction with the use of checklists is high, based on responses to the question "Do you find equipment failure checklists useful?" (1 - not at all useful to 5 - very useful). We will formally audit the time taken to diagnose equipment faults with and without checklists during simulator sessions for bank transport staff, and also plan to use these sessions to refine the checklists.

Effects of changes Our survey suggests staff feel more confident in dealing with time-critical equipment failure since checklists were introduced.

Lessons learnt The process of development of checklists highlighted variation in practice amongst staff and reinforced the need for an efficient systematic approach to be adopted. The format of "Challenge-verification-response" checklists has been tried and tested in the aviation field during time critical emergencies and we believe is transferable to a medical setting.

Message for others Although equipment failure is more critical during transport (where spares are not readily available), checklists may provide a systematic approach to other emergency situations, and could therefore be applicable in neonatal units and paediatric intensive care. However, the information contained in them must be rigorously checked and tested prior to use. In addition, the temptation to provide too much information or to provide too many checklists should be avoided, as this will dilute their effectiveness.

\section{G567(P) IMPROVING EFFICIENCY AND QUALITY OF CARE IN THE PAEDIATRIC ASSESSMENT UNIT}

NJ Creasey, G Gough, T El-Hassan, B Marden. Paediatrics, Royal United Hospital, Bath, UK

10.1136/archdischild-2015-308599.516

Context The Paediatric Assessment Unit (PAU) in our District General Hospital arose out of necessity and occupies a small space with limited privacy. Acute attendances continue to rise and demand for PAU increases.

Problem Some children wait an unacceptable time for senior paediatric review and flow through the unit is poor. Longer stays (>4-6 h) are inappropriate. Consultant input is variable and junior rotas are understaffed. Parental questionnaires stated dissatisfaction with waiting times and facilities.

Assessment of problem and analysis of its causes A working group (consultant and trainee Paediatricians, senior nurses) aimed to standardise and improve quality of care, specifically, improving waiting times and patient flow. To assess the scale of the problem we: 
1. Formally audited current practice against national standards from RCPCH's Back to Facing the Future (2013).

2. Held consultation sessions with multidisciplinary PAU team. An assessment proforma including timings, observations, history, examination and predicted outcome was designed to aid data collection.

Areas identified for improvement included:

- Consultant cover at peak times

- Facilities and space

- Waiting times to see a decision maker

- Early discharge/admission planning

- Parental information

Intervention Some improvements were more challenging to tackle than others. We are exploring relocation of PAU to a more fit for purpose area. Consultants are reviewing working patterns to provide cover during peak activity. In the meantime we carried out simpler interventions:

- Following feedback we developed the integrated assessment proforma improving assessment efficiency and focussing professionals towards planning for likely outcome. Prescription boxes and prompts will encourage early interventions (such as antipyretics, dioralyte).

- A junior doctor dedicated to PAU should meet nursing team at shift start ensuring names +/- photos are on the PAU board. A full time trust doctor employed for consistent middle grade PAU cover (although sometimes fills rota gaps).

- Consultants to attend PAU after morning ward rounds and evening handover to discuss $+/$ - review patients.

- Expected waited times standardised o Seen by doctor within 1 $\mathrm{h}$ o Seen by senior within $4 \mathrm{~h}$

- Patient/parent information leaflet distributed, outlining what to expect from a visit to PAU.

Study design Not applicable

Strategy for change We produced Standard Operating Procedures outlining the above expectations. This was presented and e-mailed to all staff. Three months were allowed for changes to be embedded before re-audit.

Measurement of improvement The results of our original and re-audit are summarised in the following Table1:

\begin{tabular}{lll} 
Abstract G567(P) Table 1 & Results of original and re-audit \\
\hline & March 2014 & July 2014 \\
& $n=72$ & $n=96$ \\
\% presenting out of hours (5 pm-9 am) & $47 \%$ & $51 \%$ \\
Seen by doctor within 1 h & $57 \%$ & $75 \%$ \\
Senior review within 4 h & $59 \%$ & $73 \%$ \\
\% of patients seen by a consultant & $12 \%$ & $23 \%$ \\
\% of admitted patients seen by consultant within 24 h & $33 \%$ & $48 \%$ \\
Correct prediction of outcome made at initial assessment & $69 \%$ & $69 \%$ \\
\hline
\end{tabular}

There was an 18\% increase in patients seen within $1 \mathrm{~h}$ and a $14 \%$ increase in patients seen by a senior within $4 \mathrm{~h}$, bringing us closer to national levels. Although consultant input doubled with our initial interventions, it is clear that planned review of consultant working patterns is necessary for us to achieve national standards.

Effects of changes These improvements led to reduced waiting times and earlier decision making and discharge from PAU. There is a tangible sense of improved parental satisfaction, although this is to be formally assessed.

Lessons learnt/Message for others To produce adequate, consistent improvement requires a longer process of workforce planning and service relocation/development. However, we have shown that significant improvements can be made by going 'back to basics'. Formalising and communicating expected standards, more efficient paperwork and maximising medical staffing, has been the mainstay to achieving improvement within our service. It can be difficult to obtain agreement for service developments from all involved, but we found involving all parties early in the process was beneficial.

Please declare any conflicts of interest below

We have no conflicts of interest to declare.

\section{G568(P) SEDATION FOR MRI/CY: A SERVICE IMPROVEMENT PROJECT}

ME Collins, BJ Shields. Paediatrics, University Hospitals Coventry and Warwickshire NHS Trust, Coventry, UK

\subsection{6/archdischild-2015-308599.517}

Context The information for this project was collected from the children's wards at UHCW. Patients attending for elective scans requiring sedation were included. Sedation was administered as per the trust protocol. This protocol is based on current NICE and $\mathrm{BNF}$ guidance.

Weight $<15 \mathrm{~kg}$ - Chloral hydrate sedation

Weight $>15 \mathrm{~kg}$ - Midazolam sedation

Problem We found that sedation of children for elective MRI/ CT scans was insufficient more often than not. This led to failed scans which in turn led to delays in diagnosis, stress for patients and families. In addition this incurred significant financial cost to the Trust due to failed scans and referrals to $\mathrm{BCH}$ for scans under GA.

Assessment of problem and analysis of its causes 60 scans were reviewed over a 6 month period in 2013. 38 children underwent midazolam sedation and 22 were given chloral hydrate.

Chloral hydrate sedation was successful in $90 \%$ of cases, whereas midazolam was only successful in $18 \%$ of cases.

After discussion with the finance department we calculated that failed scans combined with referrals to $\mathrm{BCH}$ was costing the trust c. $£ 70,000$ per year.

Intervention We have discontinued the use of midazolam sedation for MRI/CT scans

The trust is in the process of developing a paediatric GA scan service at UHCW

Strategy for change The initial results of the study were presented to the paediatric department at the monthly clinical audit meeting. After discussion, the decision to stop using midazolam for scan sedation was disseminated amongst community and hospital based paediatricians.

A multi-disciplinary team comprising paediatricians, radiographers, radiologists and anaesthetists was formed. This team is in the process of designing and trialling a GA scan service at UHCW. This will enable us to provide scans to children who would beforehand have probably had 1-2 failed scans and then been referred to $\mathrm{BCH}$. In addition this service may also provide the trust with increased revenue and savings.

Measurement of improvement We now only have children weighing $<15 \mathrm{kgs}$ (i.e. eligible for chloral sedation) attending our unit for elective CT/MRI scans. Children weighing $>15 \mathrm{kgs}$ that require sedation are being referred directly to $\mathrm{BCH}$ for GA scans while we develop our own service. This ensures that they will not need to undergo a probably unsuccessful scan under sedation with us before being referred. 